\title{
The impact of donor-specific alloantibodies on chronic antibody- mediated rejection after pediatric living donor liver transplantation
} \author{
Masaki Yamada ${ }^{2}$, Takako Yoshioka ${ }^{3}$, Mureo Kasahara ${ }^{1}$

\footnotetext{
${ }^{1}$ Organ Transplantation Center, National Center for Child Health and Development, Tokyo, Japan

${ }^{2}$ Department of Advanced Medicine for Viral Infections, National Center for Child Health and Development, Tokyo, Japan

${ }^{3}$ Department of Pathology, National Center for Child Health and Development, Tokyo, Japan
}

Seiichi Shimizu', Seisuke Sakamoto ${ }^{1}$, Akinari Fukuda ${ }^{1}$, Yusuke Yanagi ${ }^{1}$, Hajime Uchida ${ }^{1}$, Kotaro Mimori ${ }^{1}$, Toshimasa Nakao ${ }^{1}$,

Background: The development of anti-human leukocyte antigen donor-specific alloantibodies (DSA) after living donor liver transplantation (LDLT) can lead to chronic antibody-mediated rejection (CAMR). This study aimed to analyze the detail of DSA and CAMR in pediatric LDLT recipients.

Methods: We conducted a cross-sectional study for 342 pediatric recipients who were followed up for $>3$ years after primary LDLT and who had at least one screening for anti-human leukocyte antigen (HLA) antibodies. Liver biopsy was performed in the DSA positive cases with mean fluorescence intensity (MFI) $>1,000$.

Results: The median years from LDLT to DSA screening was 5.0 years (1.0-13.0 years). Of the 342 patients, 89 (26.0\%) had DSA (only class I, 11; only class II, 66; both class I and II, 12). The degree of HLA mismatch and a large amount of red blood cell (RBC) transfusion at LDLT were associated with the occurrence of DSA ( $P=0.0082$ and $P=0.0013$, respectively). Liver biopsy specimens could be scored for the 58 DSA positive recipients. cAMR was detected in 15 recipients ( $4.4 \%$ of cohort, and $25.9 \%$ of DSA positive), low graft weight at LDLT and the highest MFI were the risk factors for the occurrence of cAMR $(P=0.0094$ and $P=0.0031$, respectively), though other immunological factors or patients' characteristics were not found as risk factors for DSA and cAMR. The pathological findings after the augmentation of immunosuppression showed improvement of inflammation and C4d staining compared with before the augmentation $(P=0.027)$. In addition, MMF contributed the reduction of DSA in the cAMR cases.

Conclusions: We identified the degree of HLA mismatch and a large amount of RBC transfusion at LDLT as the risk factors of DSA development. A low graft weight and high MFI of DSA were detected as the risk factors of cAMR. Tacrolimus and MMFbased immunosuppression appeared effective for cAMR patients with high MFI. 